REAL WORLD EVIDENCE FOR ACUTE STROKE THROMBECTOMY IN THE UNITED STATES FROM THE NEUROVASCULAR QUALITY INITIATIVE-QUALITY OUTCOMES DATABASE

${ }^{1} \mathrm{R}$ Abdalla, ${ }^{1} \mathrm{D}$ Cantrell, ${ }^{2} \mathrm{M}$ Potts, ${ }^{2} \mathrm{~B}$ Jahromi, ${ }^{2} \mathrm{~A}$ Shaibani, ${ }^{2} \mathrm{M}$ Hurley, ${ }^{3} \mathrm{~S}$ Ansari ${ }^{*}$, on behalf of the NVQI-QOD Registry. ${ }^{1}$ Radiology, Northwestern University - Feinberg school of Medicine, Chicago, IL; ${ }^{2}$ Radiology, Neurosurgery, Northwestern University - Feinberg school of Medicine, Chicago, IL; ${ }^{3}$ Radiology, Neurosurgery and Neurology, Northwestern University - Feinberg school of Medicine, Chicago, IL

\subsection{6/neurintsurg-2020-SNIS.26}

Background and Purpose Multiple randomized controlled trials demonstrated the significant clinical benefit of endovascular stroke thrombectomy in anterior circulation large vessel occlusions (LVO) within 6 hours. More recent trials have shown the benefit to extend up to 24 hours with advanced imaging selection. We aim to present real-world evidence for endovascular thrombectomy (EVT) practiced in the United States using the Neurovascular Quality Initiative (NVQI) registry.

Methods Data from the NVQI registry was analyzed for anterior circulation acute ischemic strokes treated with EVT in 23 US centers (17 states) from Feb 2015 to Oct 2019.

Results Anterior circulation EVT was performed in $90.5 \%$ (2808/3103 patients). LVO sites were most common in the MCA (74\%) vs. the ICA (25\% cervical and/or terminus) with few isolated ACA occlusions (1\%). Mean age at intervention was $68.8 \pm 15.4(48.6 \%$ male $)$ presenting with a mean NIHSS of $15.8 \pm 6.8$. ASPECTS was reported in $69.2 \%$ of cases with only $47.9 \%$ presenting with ASPECTS $\geq 7$. CT/CTA was performed in $94 \%$ of cases whereas MRI/MRA was limited to $14 \%$. The median arrival to groin puncture time was $87 \mathrm{~min}$, onset to groin time was $291 \mathrm{~min}$, and procedure time was $33 \mathrm{~min}$. $43.1 \%$ of patients received IV rtPA. $84 \%$ of cases were successfully treated (TICI $2 \mathrm{~b} / 3$ reperfusion), $45 \%$ reported single pass successful recanalization, with $3.9 \%$ technical failures $(2.3 \%$ access related). Intraprocedural complications were reported in $4.6 \%$ of cases $(0.4 \%$ vessel perforations, $0.5 \%$ hemorrhagic transformations, $1.1 \%$ non-target embolization, and $1.2 \%$ iatrogenic dissections). Mean postoperative length of stay was $8.5 \pm 11.4$ days, with $11.2 \%$ inhospital mortality. 90 day follow-up mRS was reported in $67.5 \%$ of cases with mRS $\leq 2$ achieved in $37.3 \%$ and 90-day mortality reported in only $1.3 \%$ of cases. Missing data were most common in technical failure, intraprocedural complications, and 90-day mRS amounting to $13.5 \%, 15.3 \%$ and $32.5 \%$ of cases, respectively.

Conclusion Stroke thrombectomy practices, procedural metrics, and outcomes from the NVQI registry mirrors the findings from the HERMES meta-analysis confirming the real-world practice and evidence for EVT in the US. Stricter quality reporting guidelines, improving clinical follow up, and the potential for automated imaging data adjudication will transition quality reporting infrastructures to be utilized for regulatory device monitoring, expanding indications, clinical trials, and value-based reimbursements.

Disclosures R. Abdalla: None. D. Cantrell: None. M. Potts: None. B. Jahromi: None. A. Shaibani: None. M. Hurley: None. S. Ansari: None.

\section{0-027 FAVORABLE VENOUS MICROVASCULAR PROFILE IS ASSOCIATED WITH SMALLER ISCHEMIC LESION GROWTH AND SMALLER FINAL CORE INFARCTION VOLUME IN PATIENTS WITH ACUTE ISCHEMIC STROKE DUE TO LARGE VESSEL OCCLUSION}

${ }^{1} \mathrm{~T}$ Faizy*, ${ }^{1} \mathrm{R}$ Kabiri, ${ }^{1} \mathrm{M}$ Leipzig, ${ }^{2} \mathrm{G}$ Broocks, ${ }^{1} \mathrm{~S}$ Christensen, ${ }^{2} \mathrm{~F}$ Flottmann, ${ }^{3} \mathrm{M}$ Lansberg, ${ }^{3} \mathrm{G}$ Albers, ${ }^{2} \mathrm{~J}$ Fiehler, ${ }^{1} \mathrm{M}$ Wintermark, ${ }^{1} \mathrm{~J}$ Heit. ${ }^{1}$ Neuroimaging and Neurointervention, Stanford University, Stanford, CA; ${ }^{2}$ Neuroimaging and Neurointervention, University Medical Center Hamburg-Eppendorf, Hamburg, GERMANY; ${ }^{3}$ Neurology, Stanford University, Stanford, CA

\subsection{6/neurintsurg-2020-SNIS.27}

Purpose In the event of an acute ischemic stroke due to large vessel occlusion (AIS-LVO), patients with large core infarction and malignant edema have worse outcomes. Core infarction size growth is caused by poor cerebral blood flow and impaired microvascular perfusion. Cerebral microvascular perfusion is governed by the in-flow of arterial blood to the brain tissue, but also likely by the outflow of blood through the cerebral veins. Venous blood flow in the context of AISLVO may better indicate the the overall quality of tissue perfusion, as it reflects blood flow after passing the brain tissue. We determined if the venous microperfusion profile (VMP) predict ischemic lesion growth and final infarct core in AISLVO patients.

Materials and Methods We performed a multicenter, retrospective cohort study of AIS-LVO patients undergoing thrombectomy triage with CT angiography (CTA) and CT perfusion (CTP). Patients with motion artifact and incomplete electronical medical data were excluded. Patient details were obtained from prospectively maintained stroke databases and the electronic medical record. VMP was determined by opacification of the vein of Labbé, sphenoparietal sinus, and superficial middle cerebral vein on prethrombectomy CTA as: 0, not visible; 1 , moderate opacification; and 2, full. Brain edema progression and infarct growth as assessed by Net Water Uptake (NWU), which was calculated on pre-treatment and post-thrombectomy non contrast computed tomography images using manual regions of interest. Primary outcome measure was ischemic lesion growth after thrombectomy. Secondary outcome was final core infarction volume, which was manually segmented on follow-up CT and MRI studies 24-48 hours after thrombectomy.

Results 250 patients met inclusion criteria. Median patient age was 76 (IQR 65-82). 50\% were female. Linear regression models found that increased patient age $(p=0.011)$, higher blood glucose levels $(p=0.007)$, lower TICI scores $(\mathrm{p}<0.001)$ and reduced VMP $(\mathrm{p}<0.001)$ predicted increased core infarction growth (higher NWU). In a multivariate regression analysis, poor VMP predicted core infarct growth while controlling for age, blood glucose, and TICI score $(\beta=-2.111840, \quad[\quad 95 \% \quad C I-2.808059256-1.41562150]$; $\mathrm{p}<001)$. In a secondary analysis focused on final infarct core, we excluded 5 patients due to missing final infarct size data. After controlling for age, blood glucose, and TICI score, poor VMP predicted higher final core infarct size $\left(\beta=-22.57626,\left[\begin{array}{llll}95 \% & \text { CI } & -30.7799325 & -14.3725813\end{array}\right]\right.$; $\mathrm{p}<001)$. 
Conclusion Poor cerebral perfusion on the venous microvascular profile predicts ischemic lesion growth and final infarct core volume in AIS-LVO patients treated with thrombectomy. Disclosures T. Faizy: None. R. Kabiri: None. M. Leipzig: None. G. Broocks: None. S. Christensen: None. F. Flottmann: None. M. Lansberg: None. G. Albers: None. J. Fiehler: None. M. Wintermark: None. J. Heit: None.

\section{0-028 THE INTRASACCULAR SEAL ${ }^{\odot}$ DEVICE: IMPROVED FLEXIBILITY AND HEALING}

${ }^{1} \mathrm{~A}$ Kraitem, ${ }^{1} \mathrm{M}$ Gounis*, ${ }^{*} \mathrm{Z}$ Vardar, ${ }^{1} \mathrm{R}$ King, ${ }^{1} \mathrm{E}$ Langan, ${ }^{2} \mathrm{~T}$ Wolfe, ${ }^{3} \mathrm{~A}$ Badruddin, ${ }^{4}$ E Periera, ${ }^{5} \mathrm{~B}$ Follmer, ${ }^{5} \mathrm{~A}$ Rosqueta, ${ }^{6} \mathrm{O}$ Zaidat. ${ }^{1}$ Radiology, University of Massachusetts, Worcester, MA; ${ }^{2}$ Aurora Neuroscience Innovation Institute, Aurora Health, Milwaukee, WI; ${ }^{3}$ Neurology, Community Hospital, Munster, IN; ${ }^{4}$ Vascular and Interventional Radiology, Biscayne Medical Arts Center, Miami, FL; ${ }^{5}$ RandD, Galaxy Therapeutics, Broofield, Wl; ${ }^{6}$ Endovascular Neurosurgery and Stroke, St Vincent Medical Center, Toledo, $\mathrm{OH}$

10.1136/neurintsurg-2020-SNIS.28

Background The new generation of intrasaccular flow disruptors offers several potential advantages in aneurysm treatment. ${ }^{1}{ }^{2}$ However, questions regarding risks for thromboembolic complications ${ }^{34}$ and long-term treatment durability remain. We study the safety and effectiveness of three Galaxy Therapeutics Seal $\subset$ intrasaccular prototypes: A, B, and C (Galaxy Therapeutics LLC, Brookfield, WI).

Methods Aneurysms were created in thirteen rabbits and one of 3 Seal devices were implanted. High frequency optical coherence tomography (HF-OCT) and Digital Subtraction Angiography (DSA) was performed 4- and 12-weeks postimplant. After 12 weeks, the parent vessel and the aneurysm were explanted for histological analysis. One rabbit developed symptoms consistent with a lumbar spine injury and was euthanized and explanted at day 56.

Results 12-week DSA and OCT images demonstrated 8 animals had a satisfactory aneurysm occlusion (group 1: complete blood flow disruption or contrast filling in marker recess) and 4 into group 2 (residual neck or aneurysm). The percentage of neointimal coverage of the device at four-weeks was greater for group $1(78 \pm 22.6 \%)$ than for group $2(37 \pm 4.7 \%)$ $(p=0.006)$ which persisted at 12 -weeks $(87.5 \pm 14 \%$ vs. 41 $\pm 3.1 \%$ respectively; $p=0.004)$. There was no statistical difference between groups 1 and 2 in either baseline neck gap areas $\left(0.8 \pm 0.64 \mathrm{~mm}^{2}\right.$ vs. $2.6 \pm 1.42 \mathrm{~mm}^{2}$, respectively; $p=0.808)$ nor in baseline neck gap volumes $\left(26 \pm 26 \mathrm{~mm}^{3}\right.$ vs. $113.11 \pm 134.17 \mathrm{~mm}^{3}$, respectively; $p=0.361$ ). Representative examples of pathology are shown in the figure 1 .

Conclusion Preliminary evidence in this preclinical study highlight advantages of a new generation of intrasaccular aneurysm embolization technology in terms of flexibility and optimization of healing features, particularly in the $\mathrm{A}$ and $\mathrm{C}$ cohorts.

\section{REFERENCES}

1. Radiology 2014;273:194-201.

2. I Neurointerv Surg 2019:11:1150-1154

3. World Neurosurg 2018;109:e183-e193.

4. J Neurointerv Surg 2018;10:553-559.

Disclosures A. Kraitem: None. M. Gounis: 1; C; Research grant to institution to fund experiments by Galaxy Therapeutics. Z. Vardar: None. R. King: None. E. Langan: None. T. Wolfe: 4; C; Founder, Galaxy Therapeutics. A. Badruddin: 4; C; Founder, Galaxy Therapeutics. E. Periera: 4; C; Founder, Galaxy Therapeutics. B. Follmer: 5; C; Galaxy Therapeutics. A. Rosqueta: 5; C; Galaxy Therapeutics. O. Zaidat: 4; C; Founder, Galaxy Therapeutics.

\section{0-029 OUTCOMES OF ENDOVASCULAR TREATMENT OF VEIN OF GALEN ANEURYSMAL MALFORMATION IN NEONATES}

P Seleznev, K Orlov*, V Berestov, A Gorbatykh, D Kislitsyn, T Shayakhmetov, N Strelnikov. Meshalkin National Medical Research Center, Novosibirsk, Russian Federation

\subsection{6/neurintsurg-2020-SNIS.29}

Objective Vein of Galen aneurysmal malformation is a rare congenital cerebrovascular disorder whose natural course has almost no favorable outcomes $(<1 \%)$. Our objective was to analyze the outcomes of endovascular treatment of patients with vein of Galen aneurysmal malformation (VGM) during the first days of their life.

Materials and Methods During the period between January 2013 and February 2020, 52 patients with vein of Galen malformations were operated on at the National Medical Research Center named after Academician E.A. Meshalkin. Eleven of these patients were younger than 10 days when surgery was performed. Twelve endovascular embolization sessions were conducted via a transarterial approach. Cyanacrylate glue was used in 10 cases (mural VGMs); in one case, Phil 25 non-adhesive liquid embolic system was used and two embolization stages were performed (choroidal VGM). All patients were hospitalized in an intensive care unit having the Bicêtre score of 1012. Prior to surgery, all patients underwent cardiac ultrasonography (that involved measuring pulmonary artery pressure) and neurosonography that involved measuring the linear blood flow velocity (LBFV) in the vein of Galen and afferent vessels. All patients had pronounced pulmonary hypertension; LBFV in the vein of Galen was $\geq 0.6 \mathrm{~m} / \mathrm{s}$.
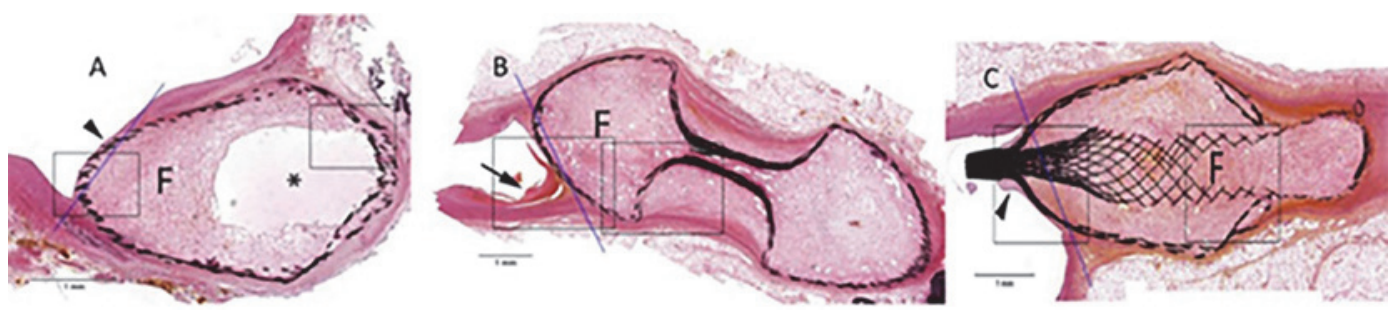

Abstract 0-028 Figure 1 H\&E stains for the three prototypes (A), (B), and (C) 12-weeks following implant. Solid line=approximate aneurysm neck. Arrowhead = mature and stable neointima covering device; $F=$ fibrovascular tissue partially filling the fundus; $A$ : asterisk = sequestered cystic space within the fundus (not connected to parent vessel). B: arrow=fibrin thrombus in parent artery recess at neck 